\title{
Molecular Typing Reveals Frequent Clustering among Human Isolates of Listeria monocytogenes in Italy
}

\author{
CATERINA MAMMINA, ${ }^{1 *}$ GERARDO MANFREDA, ${ }^{2}$ AURORA ALEO,${ }^{1}$ ALESSANDRA DE CESARE, ${ }^{2}$ \\ NATHALIE PELLISSIER, ${ }^{3}$ CRISTINA ROMANI, ${ }^{4}$ PIERLUIGI NICOLETTI, ${ }^{5}$ PATRIZIA PECILE, ${ }^{5}$ \\ ANTONINO NASTASI, ${ }^{4}$ AND MIRELLA M. PONTELLO ${ }^{3}$
}

\begin{abstract}
${ }^{1}$ Department of Sciences for Health Promotion “G. D'Alessandro," University of Palermo, Via del Vespro 133, I-90127 Palermo, Italy; ${ }^{2}$ Department of Food Science, Alma Mater Studiorum University, Viale Fanin 44, I-40127 Bologna, Italy; ${ }^{3}$ Department of Public Health, Microbiology and Virology, University of Milan, Via C. Pascal n. 36, I-20133 Milan, Italy; ${ }^{4}$ Department of Public Health, University of Florence, Viale G. B. Morgagni 48, I-50134 Florence, Italy; and ${ }^{5}$ Laboratory of Microbiology and Virology, Azienda Ospedaliero-Universitaria Careggi, Viale G. B. Morgagni 85, I-50134 Florence, Italy
\end{abstract}

MS 08-453: Received 12 September 2008/Accepted 18 November 2008

\begin{abstract}
In Italy, the annual incidence of reported cases of listeriosis amounts in recent years (2004 to 2006) to 0.8 case per million inhabitants. Our study is a subtyping analysis by serotyping, ribotyping, and pulsed-field gel electrophoresis analysis of 44 human isolates from apparently sporadic cases of infection in the Lombardy region and in the Province of Florence, Italy, in the years 1996 to 2007. Based on the results of the different subtyping methods, 10 occasions were detected when strains of L. monocytogenes with the same subtype were isolated from more than one listeriosis case. A total of 28 (66.7\%) of 44 isolates were attributed to molecular subtype clusters. Our data support the use of sensitive molecular approaches to identify and trace L. monocytogenes isolates responsible for foodborne outbreaks of human listeriosis.
\end{abstract}

Listeria monocytogenes is the etiological agent of listeriosis, a foodborne disease affecting humans and a variety of vertebrates (23). Listeriosis occurs primarily in immunocompromised individuals, causing septicemia and central nervous system infections, and in pregnant women who may suffer from preterm delivery, miscarriage, or stillbirth (21). Healthy adults may show afebrile gastroenteritis after ingesting a large number of L. monocytogenes cells (16).

Most industrialized countries, including those within the European Union, have an annual incidence of listeriosis between 2 and 10 reported cases per million people per year (22). However, listeriosis has one of the highest case fatality rates (approximately 20\%) among foodborne diseases (21).

In Italy, notification of listeriosis cases has been mandatory since 1993, but transmission of data to the national level is done quarterly. A second syndrome-based surveillance system is in place, which covers infections of the central nervous system. A national reference laboratory, which receives strains and epidemiological and clinical information on a voluntary basis, is also present. However, the incidence of reported cases by the Ministry of Health is lower than most European Union countries, amounting in recent years (2004 to 2006) to 0.8 case per million inhabitants annually (10). This low incidence must be considered with care because of poor sensitivity of the passive surveillance systems and the diagnostic difficulties posed

* Author for correspondence. Tel: +39 0916553623; Fax: +39 0916553641; E-mail: diptigmi@unipa.it. by $L$. monocytogenes infections, the long incubation period (7 to 60 days), the typical occurrence in specific risk groups (pregnant women, newborns, and persons who are immunocompromised), and the association to different clinical presentations (22). Indeed, a recent report shows an incidence of 1.3 cases per $1,000,000$ inhabitants during a 1year (2002 to 2003) period of laboratory-based surveillance (5). Moreover, a reinforcement of the surveillance system put in place in 2006, in the Lombardy region, combining collaborative epidemiological investigations, use of subtyping methods for cluster identification, and application of the capture-recapture method to estimate incidence, has given an estimated annual incidence equal to 6.6 per million inhabitants (17).

Throughout Europe and the United States, the majority of cases of listeriosis reported to public health authorities is apparently not linked to a common source and is therefore defined as "sporadic" (20). However, because of the unique epidemiological and clinical characteristics of human foodborne listeriosis, traditional epidemiologic surveillance systems alone are unable to detect most common source outbreaks, particularly when a limited number of cases are scattered over a wide geographic area $(18,19)$. Therefore, use of effective subtyping methods, including serotyping as well as different DNA-based subtyping methods-ribotyping, pulsed-field gel electrophoresis (PFGE), PCR, restriction fragment length polymorphism (RFLP) analysis, and multilocus sequence typing-is essential for 
$\begin{array}{lllllllllllllllllllllllllllll}\text { MW } & 1 & 2 & 3 & 4 & 5 & 6 & 7 & 8 & 9 & 10 & 11 & 12 & 13 & 14 & 15 & 16 & 17 & 18 & 19 & 20 & 21 & 22 & 23 & 24 & 25 & 26 & 27 & 28\end{array}$

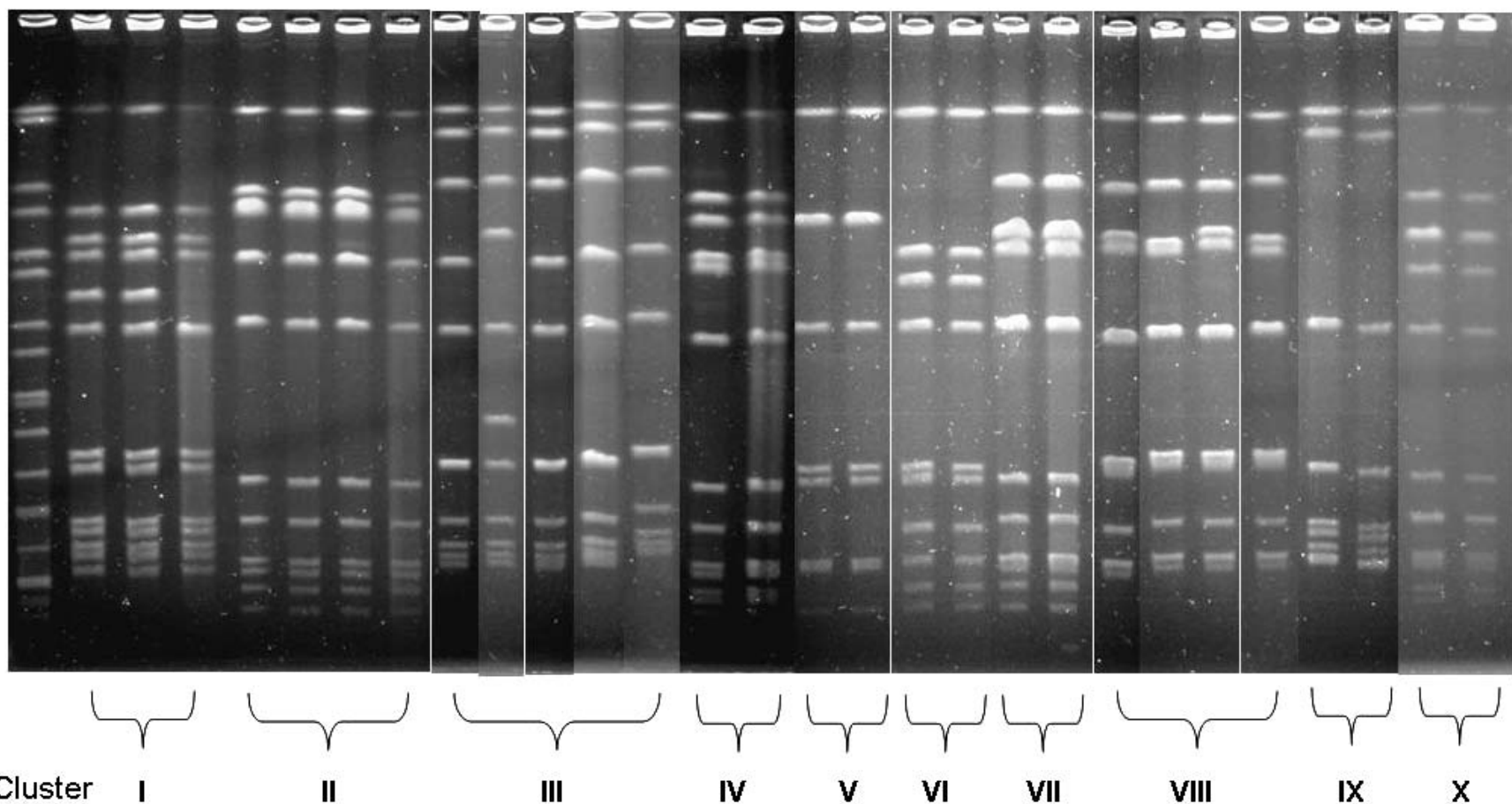

FIGURE 1. PFGE typing after digestion by AscI of clustered strains of Listeria monocytogenes isolated in Lombardy and Tuscany, Italy. Lane MW, Xbal-digested DNA of Salmonella enterica serotype Braenderup H9812; lanes 1 through 28, isolates belonging to clusters I to X (strain designation, place and time of isolation, and phenotypic and genetic properties are shown in the Table 1).

the epidemiological investigations of $L$. monocytogenes $(12,18,19,24)$.

Our study is a retrospective subtyping analysis of 32 human isolates identified from apparently sporadic cases of infection in the Lombardy region and in the Province of Florence, Italy, in the years 2006 to 2007. Twelve further isolates from human cases occurring in the same geographic areas since 1996 were available for the study. The objective was to detect clustering of isolates by using serotyping and DNA-based subtyping methods, and to evaluate contribution of the molecular subtyping approach to identification of possible common source outbreaks.

\section{MATERIALS AND METHODS}

Bacterial isolates. Between January 2006 and July 2007, 24 human isolates from cases of listeriosis were collected from the laboratories of diagnostic microbiology of the Lombardy region, Italy. Eight further isolates were recovered in the same period from the Careggi Hospital of Florence, Tuscany, Italy. Twelve isolates of L. monocytogenes from human sources identified by the same laboratories during the previous years were also collected. These geographical areas were chosen based on the willingness of the regional health authorities of Lombardy and the laboratory microbiologists of the Careggi Hospital of Florence to participate in a collaborative study on L. monocytogenes epidemiology. In Lombardy, a strengthening of the surveillance system was implemented in the period of the study.

The strains were isolated from blood or from cerebrospinal fluid, mostly from immunocompromised or elderly patients. Only three strains were from maternal-fetal infections. Each isolate in this study represents a single human listeriosis case. No case had been formally recognized as belonging to a foodborne outbreak or associated to a specific food vehicle.
The International Life Sciences Institute North America $L$. monocytogenes outbreak and diversity sets were used as reference strains (4).

Serotyping. All strains tested were serotyped by following the manufacturer's instructions by using commercial specific antisera (Denka Seiken, Tokyo, Japan).

Ribotyping. Isolates were characterized by automated ribotyping by using the RiboPrinter (DuPont Qualicon, Inc., Wilmington, DE), as described elsewhere (1). EcoRI was selected as the restriction enzyme. The automatic classification into ribotypes performed by the RiboPrinter software was the first criterion to cluster the strains into ribotypes. However, some strains underwent manual classification after visual inspection of the profile; a single, clear band was considered difference enough to distinguish ribotypes.

PFGE analysis. PFGE was performed according to the PulseNet protocol with enzymes AscI and ApaI (7). Bacterial cultures were embedded in agarose, lysed, washed, digested with the restriction enzyme, and electrophoresed on a Chef Mapper XA (Bio-Rad Laboratories, Hercules, CA) at $6 \mathrm{~V} / \mathrm{cm}$ for $22 \mathrm{~h}$, with switch times of 4 to 40.01 s. XbaI-digested DNA of Salmonella enterica serotype Braenderup H9812 was used as size reference standard (7). PFGE patterns were compared by means of the Dice coefficient by using the fingerprinting Molecular Analyst software (Bio-Rad Laboratories). The results of the clustering analysis were confirmed by visual comparison of the PFGE profiles. In a first round of typing, AscI was used. Two profiles obtained by AscI were classified as "indistinguishable" when the DNA fragment patterns matched each other completely, "closely related" if they differed by one to three bands, or "unrelated" if they differed by more than three bands. Numbers were used to designate the AscI profiles. Closely related patterns were assigned an additional cap- 
TABLE 1. Characteristics of clustered isolates of Listeria monocytogenes in Italy

\begin{tabular}{|c|c|c|c|c|c|c|c|c|c|}
\hline \multirow[b]{2}{*}{ Lane } & \multirow[b]{2}{*}{ Isolate } & \multirow[b]{2}{*}{ Isolation place } & \multirow[b]{2}{*}{ Isolation time } & \multirow[b]{2}{*}{ Serotype } & \multicolumn{5}{|c|}{ AscI PFGE } \\
\hline & & & & & Ribotype & DUP-ID & type & Lineage & Cluster \\
\hline 1 & LMOH-MI-1 & Lodi, Lombardy & 6 July 2006 & $1 / 2 \mathrm{~b}$ & $309 \mathrm{~S} 2$ & 1034 & $1 \mathrm{~A}$ & I & I \\
\hline 2 & LMOH-MI-7 & Brescia, Lombardy & 11 May 2006 & $1 / 2 \mathrm{~b}$ & $309 \mathrm{~S} 2$ & 1034 & $1 \mathrm{~A}$ & I & \\
\hline 3 & LMOH-MI-11 & Milan, Lombardy & 20 October 2006 & $1 / 2 \mathrm{~b}$ & $356 \mathrm{~S} 3$ & 1042 & 1B & I & \\
\hline 4 & LMOH-MI-3 & Bergamo, Lombardy & 2 August 2006 & $1 / 2 \mathrm{a}$ & 204 S5 & 1045 & 2 & II & II \\
\hline 5 & LMOH-MI-19 & Bergamo, Lombardy & 21 January 2007 & $1 / 2 \mathrm{a}$ & 204 S5 & 1045 & 2 & II & \\
\hline 6 & LMOH-MI-21 & Bergamo, Lombardy & 21 February 2007 & $1 / 2 \mathrm{a}$ & 204 S5 & 1045 & 2 & II & \\
\hline 7 & LMOH-MI-25 & Lodi, Lombardy & 30 April 2007 & $1 / 2 \mathrm{a}$ & 204 S5 & 1045 & 2 & II & \\
\hline 8 & LMOH-MI-13 & Milan, Lombardy & 17 November 2006 & $4 b$ & $423 \mathrm{~S} 6$ & 1044 & 3 & I & III \\
\hline 9 & LMOH-MI-20 & Milan, Lombardy & 19 January 2007 & $4 b$ & $423 \mathrm{~S} 6$ & 1044 & 3 & I & \\
\hline 10 & LMOH-FL-21 & Florence, Tuscany & 26 January 2007 & $4 b$ & $423 \mathrm{~S} 6$ & 1044 & 3 & I & \\
\hline 11 & LMOH-FL-31 & Florence, Tuscany & 26 January 2007 & $4 b$ & $423 \mathrm{~S} 6$ & 1044 & 3 & I & \\
\hline 12 & LMOH-MI-27 & Milan, Lombardy & 8 June 2007 & $4 \mathrm{~b}$ & $423 \mathrm{~S} 6$ & 1044 & 3 & I & \\
\hline 13 & LMOH-MI-16 & Milan, Lombardy & 12 January 1996 & $1 / 2 \mathrm{a}$ & $303 \mathrm{~S} 5$ & 18603 & 4 & II & IV \\
\hline 14 & LMOH-FL-26 & Florence, Tuscany & 27 July 2005 & $1 / 2 \mathrm{a}$ & $303 \mathrm{~S} 5$ & 18603 & 4 & II & \\
\hline 15 & LMOH-MI-17 & Bergamo, Lombardy & 11 December 2006 & $1 / 2 \mathrm{a}$ & $303 \mathrm{~S} 1$ & 18613 & 5 & II & $\mathrm{V}$ \\
\hline 16 & LMOH-MI-18 & Bergamo, Lombardy & 8 December 2006 & $1 / 2 \mathrm{a}$ & $303 \mathrm{~S} 1$ & 18613 & 5 & II & \\
\hline 17 & LMOH-FL-28 & Florence, Tuscany & 27 July 2006 & $1 / 2 \mathrm{a}$ & $347 \mathrm{~S} 1$ & 18598 & 7 & II & VI \\
\hline 18 & LMOH-FL-20 & Florence, Tuscany & 2 August 2006 & $1 / 2 \mathrm{a}$ & $347 \mathrm{~S} 1$ & 18598 & 7 & II & \\
\hline 19 & LMOH-FL-24 & Florence, Tuscany & 19 March 2005 & $1 / 2 \mathrm{a}$ & $303 \mathrm{~S} 5$ & 18603 & 8 & II & VII \\
\hline 20 & LMOH-FL-25 & Florence, Tuscany & 19 March 2005 & $1 / 2 \mathrm{a}$ & $303 \mathrm{~S} 5$ & 18603 & 8 & II & \\
\hline 21 & LMOH-FL-18 & Florence, Tuscany & 25 July 2005 & $4 b$ & $355 \mathrm{~S} 4$ & 1038 & $6 \mathrm{~B}$ & I & VIII \\
\hline 22 & LMOH-FL-19 & Florence, Tuscany & 27 July 2005 & $4 b$ & $355 \mathrm{~S} 4$ & 1038 & $6 \mathrm{~A}$ & I & \\
\hline 23 & LMOH-MI-4 & Como, Lombardy & 26 June 2007 & $4 \mathrm{~b}$ & $355 \mathrm{~S} 4$ & 1038 & $6 \mathrm{~A}$ & I & \\
\hline 24 & LMOH-MI-28 & Milan, Lombardy & 8 June 2007 & $4 \mathrm{~b}$ & $355 \mathrm{~S} 4$ & 1038 & $6 \mathrm{~A}$ & I & \\
\hline 25 & LMOH-MI-9 & Brescia, Lombardy & 19 July 2006 & $4 b$ & $356 \mathrm{~S} 1$ & 18624 & 22 & I & IX \\
\hline 26 & LMOH-MI-14 & Milan, Lombardy & 16 November 2006 & $4 \mathrm{~b}$ & $356 \mathrm{~S} 1$ & 18624 & 22 & I & \\
\hline 27 & LMOH-MI-23 & Lodi, Lombardy & 11 October 2000 & $1 / 2 \mathrm{a}$ & $202 \mathrm{~S} 1$ & 19171 & 23 & II & $\mathrm{X}$ \\
\hline 28 & LMOH-MI-26 & Milan, Lombardy & 30 April 2007 & $1 / 2 \mathrm{a}$ & $202 \mathrm{~S} 1$ & 19171 & 23 & II & \\
\hline
\end{tabular}

ital letter. Indistinguishable or closely related strains were subsequently cleaved with ApaI.

PCR-RFLP of virulence genes. For all isolates, the $L$. monocytogenes virulence genes encoding listeriolysin $\mathrm{O}$ (hly), actA ( $a c t A)$, and internalin A (inlA) were PCR amplified, and hly and inlA amplicons screened for allelic polymorphisms by PCRRFLP according to Wiedmann et al. (25). PCR products were digested separately by using HhaI and HpaII for hly, and ApoI, HinfI, and HhaI for inlA. For actA, PCR amplification of the proline-rich region, with primers PR5 and PR3 was performed according to Inoue et al. (9). Two alleles, characterized by the presence of one proline-rich unit (518-bp amplification product) or two (623-bp amplification product), were classified as actA type II and actA type I, respectively. Different combinations of allelic polymorphisms were assigned arbitrary small letters.

Genetic lineage analysis. Isolates were assigned to lineages I and II based on serotype, DuPont identification pattern number (DUP-ID), and PCR-RFLP of virulence genes according to Wiedmann et al. (25).

Simpson's index of discrimination (SID). The suitability of serotyping, ribotyping, PFGE, and PCR-RFLP for differentiation of L. monocytogenes human isolates included in this study was determined by using SID, as described by Hunter and Gaston (8).

\section{RESULTS}

Serotyping. A total of $20(46 \%)$ clinical isolates were classified as serotype $1 / 2 \mathrm{a}, 19(43 \%)$ as serotype $4 \mathrm{~b}$, and 5 (11\%) as serotype $1 / 2 \mathrm{~b}$ (Table 1 ).
Ribotyping. A total of 17 different ribotypes, characterized by eight or nine bands with a molecular mass ranging between 2.1 and $48 \mathrm{kbp}$, were identified among the 44 human strains. The similarity between these profiles ranged between 49 and $92 \%$. Overall, 5 ribotypes were associated to single strains, whereas 12 ribotypes were shared by two to six isolates (Table 1).

Fourteen different DUP-IDs were assigned to the isolates tested (Table 1). Among the identified DUP-IDs, 1034, 1038, 1042, 1044, and 1052 are classified in lineage I, whereas DUP-IDs 1045 and 1047 are classified in lineage II (25).

PFGE. Twenty-six AscI pulsotypes were recognized among the 44 human isolates. Unique AscI pulsotypes were demonstrated by 16 isolates, whereas eight indistinguishable and two closely related pulsotypes were shared by 2 to 5 isolates (Fig. 1 and Table 1). ApaI profiles of the strains with indistinguishable AscI profiles showed no difference in number or position of bands in seven out of eight cases, whereas in one case, the ApaI profile of one isolate differed by two bands. Closely related isolates by AscI differed by one to three bands by ApaI as well.

PCR-RFLP. PCR-RFLP of virulence genes actA, hly, and inlA identified nine different combinations of restriction patterns. Except for one pattern associated to a single strain, all PCR-RFLP patterns were shared between 2 and 11 strains. 
SID. The SID values of PFGE, EcoRI ribotyping, and PCR-RFLP for the clinical isolates of this study were 0.972 , 0.941 , and 0.852 , respectively.

Clustering of $L$. monocytogenes isolates. Combining the results of PFGE and ribotyping, 10 different clusters were identified. PFGE and ribotyping yielded consistent results except for two cases, (i) cluster I, which included two isolates with indistinguishable PFGE patterns and ribotypes, and one isolate with a closely related PFGE pattern and a different ribotype, and (ii) two additional clusters, IV and VII, consisting of isolates with unrelated PFGE patterns, but identical ribotype (Table 1). Overall, a total of 28 $(66.7 \%)$ of 44 isolates were attributed to molecular subtype clusters. The number of infected people in each cluster ranged from two to a maximum of five cases. Seven clusters included isolates from the Lombardy region or, alternatively, from the Province of Florence, whereas three clusters contained isolates from the two different geographic areas. With the exception of clusters IV, VIII, and X, the strains of L. monocytogenes within a single cluster had been isolated through an interval of time ranging from a minimum of 0 days (same day) to a maximum of 14 months. Cluster IV grouped together isolates identified in the years 1996 and 2005, cluster VIII in the years 2005 and 2007, and cluster X in the years 2000 and 2007 (Table 1).

\section{DISCUSSION}

This article describes phenotypic and genetic profiles of isolates of $L$. monocytogenes obtained from two different geographic areas of Italy, which show relatively high incidence rates of listeriosis (in the years 2005 and 2006, the incidence of notifiable cases was 1.4 and 4.5 per million inhabitants in Lombardy and Tuscany, respectively).

Twenty-three and 21 human isolates tested in this study belonged to lineages I and II, respectively, in contrast to most previous literature reports in which lineage I strains were much more common among human listeriosis cases than were lineage II strains $(24,25)$. However, a recent study conducted in Italy shows, accordingly, that $44 \%$ of the strains isolated from listeriosis cases in 2002 to 2003 in Italy belonged to serotype $1 / 2$ a, typically associated with lineage II (5). Consistently, some European countries, such as Finland, report that serotype 1/2a is apparently replacing serotype $4 \mathrm{~b}$ as the serotype recovered most frequently from human infections (13).

Retrospective subtyping analysis of the 44 human $L$. monocytogenes isolates from two different geographic of Italy reveals that a large proportion of listeriosis cases can be grouped into subtype clusters, some or all of which could represent common source outbreaks. Hence, although most human listeriosis infections in Italy are being generally considered to represent sporadic cases, our results suggest that such clusters could be more common than previously assumed $(3,5,6)$.

The SID values of PFGE, EcoRI ribotyping, and PCRRFLP for the clinical isolates of this study were 0.972 , 0.941 , and 0.852 , respectively, confirming the highest discriminatory ability of PFGE. Therefore, although ribotyp- ing provides an automated, highly discriminatory, and quick (approximately $8 \mathrm{~h}$ of run time) tool able to detect putative single-source disease clusters, in public health investigations, PFGE typically provides higher sensitivity for identifying differences in molecular subtypes.

The subtype clusters reported here include both singleregion and multiregional clusters, according to previous reports documenting that human listeriosis clusters can be geographically widely distributed $(2,14)$. Specifically, cluster I, including human isolates from Lombardy, proved retrospectively to contain isolates indistinguishable by serotyping and DNA-based methods from $L$. monocytogenes strains DUP-1034 previously detected on the rinds of Taleggio cheeses produced in an Italian plant (1). Although no specific epidemiologic investigations were conducted to confirm this cluster, our findings indicate that aggressive foodborne disease-surveillance efforts would allow for a timely detection of a possible foodborne outbreak, which could in turn minimize the health and economic impact of listeriosis.

Unlikely did the eight cluster grouped strains separate by a few days to months as to their time of isolation; three clusters (IV, VIII and X) included strains isolated over intervals of 9,2 , and 7 years, respectively. A first obvious consideration about the occurrence only by chance of two indistinguishable pheno- and genotypes cannot be ignored, even though, based on the large heterogeneity of circulating pulsotypes, a coincidental episode should be unlikely. Alternative hypothesis about these long-lasting clusters should consider the year-long persistence of L. monocytogenes colonization in food processing plants, the delayed shelf lives of some food products, and the combined effects of these two events $(11,15)$.

This study has some limits. Because of the restricted geographical source of L. monocytogenes strains, a generalization of results is questionable. Moreover, an overestimate of clustering could arise from the inclusion of closely related strains. However, according with the considerations of Sauders et al. (18), for the purpose of detecting a possible outbreak, the use of more stringent criteria of inclusion, i.e., completely identical banding pattern, could miss some clusters.

From a public health point of view, a more sensitive, subtyping-based first step that is followed by a more discriminative, epidemiologically based second step appears to be the more effective approach to detection of a foodborne outbreak of listeriosis, considering the peculiar characteristics of the causal organism, most food vehicles, and the susceptible people and the consequent atypical appearance of epidemic events within a community. Although further and more extensive epidemiological studies are needed, our observations are consistent with the hypothesis that a considerable number of listeriosis cases might occur in clusters, many of which could represent single-source outbreaks liable to go undetected without the combined use of molecular subtyping methods and more aggressive and timely epidemiological investigations. 


\section{ACKNOWLEDGMENTS}

We are grateful to Dr. Martin Wiedmann, Cornell University, Ithaca, NY, for providing us the diversity and outbreak strain sets of L. monocytogenes. We also thank the personnel of the hospital microbiological laboratories of Lombardy for facilitating collection of isolates and information that served as the basis for this study. This work has been supported by a fund from the Italian University Minister, Programmi di ricerca di interesse nazionale, year 2005.

\section{REFERENCES}

1. De Cesare, A., G. Manfreda, M. Macrì, and C. Cantoni. 2007. Application of automated ribotyping to support the evaluation of Listeria monocytogenes sources in a Taleggio cheese producing plant. J. Food Prot. 70:1116-1121.

2. de Valk, H., V. Vaillant, C. Jacquet, J. Rocourt, F. Le Querrec, F. Stainer, N. Quelquejeu, O. Pierre, V. Pierre, J. C. Desenclos, and V. Goulet. 2001. Two consecutive nationwide outbreaks of listeriosis in France, October 1999-February 2000. Am. J. Epidemiol. 154:944950.

3. Franciosa, G., C. Scalfaro, A. Maugliani, F. Floridi, A. Gattuso, S. Hodzic, and P. Aureli. 2007. Distribution of epidemic clonal genetic markers among Listeria monocytogenes $4 \mathrm{~b}$ isolates. J. Food Prot. 70:574-581.

4. Fugett, E., E. Fortes, C. Nnoka, and M. Wiedmann. 2006. International Life Sciences Institute North America Listeria monocytogenes strain collection: development of standard Listeria monocytogenes strain sets for research and validation studies. J. Food Prot. 69: 2929-2938.

5. Gianfranceschi, M., A. Gattuso, M. D’Ottavio, S. Fokas, and P. Aureli. 2007. Results of a 12-month long enhanced surveillance of listeriosis in Italy. Euro Surveill. 12:E7-E8.

6. Gianfranceschi, M., A. Gattuso, S. Tartaro, and P. Aureli. 2003. Incidence of Listeria monocytogenes in food and environmental samples in Italy between 1990 and 1999: serotype distribution in food, environmental and clinical samples. Eur. J. Epidemiol. 18:10011006.

7. Graves, L. M., and B. Swaminathan. 2001. PulseNet standardized protocol for subtyping Listeria monocytogenes by macrorestriction and pulsed-field gel electrophoresis. Int. J. Food Microbiol. 65:5562 .

8. Hunter, P. R., and M. A. Gaston. 1988. Numerical index of the discriminatory ability of typing systems: an application of Simpson's index of diversity. J Clin Microbiol. 26:2465-2466.

9. Inoue, S., K. Katagiri, M. Terao, and T. Maruyama. 2001. RAPDand actA gene-typing of Listeria monocytogenes isolates of human listeriosis, the intestinal contents of cows and beef. Microbiol. Immunol. 45:127-133.

10. Istituto Superiore di Sanità. 2005. Data about surveillance of notifiable infectious diseases. Available at: http://www.simi.iss.it/ banca_dati_simi.htm. Accessed 3 November 2008.

11. Keto-Timonen, R., R. Tolvanen, J. Lundén, and H. Korkeala. 2007. An 8-year surveillance of the diversity and persistence of Listeria monocytogenes in a chilled food processing plant analyzed by amplified fragment length polymorphism. J. Food Prot. 70:1866-1873.

12. Liu, D. 2006. Identification, subtyping and virulence determination of Listeria monocytogenes, an important foodborne pathogen. $J$. Med. Microbiol. 55:645-659.

13. Lukinmaa, S., M. Miettinen, U. M. Nakari, H. Korkeala, and A. Siitonen. 2003. Listeria monocytogenes isolates from invasive infections: variation of sero- and genotypes during an 11-year period in Finland. J. Clin. Microbiol. 41:1694-1700.

14. Mead, P. S., E. F. Dunne, L. Graves, M. Wiedmann, M. Patrick, S. Hunter, E. Salehi, F. Mostashari, A. Craig, P. Mshar, T. Bannerman, B. D. Sauders, P. Hayes, W. Dewitt, P. Sparling, P. Griffin, D. Morse, L. Slutsker, B. Swaminathan, and the Listeria Outbreak Working Group. 2006. Nationwide outbreak of listeriosis due to contaminated meat. Epidemiol. Infect. 134:744-751.

15. Olsen, S. J., M. Patrick, S. B. Hunter, V. Reddy, L. Kornstein, W R. MacKenzie, K. Lane, S. Bidol, G. A. Stoltman, D. M. Frye, I. Lee, S. Hurd, T. F. Jones, T. N. LaPorte, W. Dewitt, L. Graves, M. Wiedmann, D. J. Schoonmaker-Bopp, A. J. Huang, C. Vincent, A. Bugenhagen, J. Corby, E. R. Carloni, M. E. Holcomb, R. F. Woron, S. M. Zansky, G. Dowdle, F. Smith, S. Ahrabi-Fard, A. R. Ong, N. Tucker, N. A. Hynes, and P. Mead. 2005. Multistate outbreak of Listeria monocytogenes infection linked to delicatessen turkey meat. Clin. Infect. Dis. 40:962-967.

16. Ooi, S. T., and Lorber B. 2005. Gastroenteritis due to Listeria monocytogenes. Clin. Infect. Dis. 40:1327-1332.

17. Pontello, M. (University of Milan). 2008. Personal communication.

18. Sauders, B. D., E. D. Fortes, D. L. Morse, N. Dumas, J. A. Kiehlbauch, Y. Schukken, J. R. Hibbs, and M. Wiedmann. 2003. Molecular subtyping to detect human listeriosis clusters. Emerg. Infect. Dis. 9:672-680.

19. Sauders, B., D., Y, Schukken, L. Kornstein, V. Reddy, T. Bannerman, E. Salehi, N. Dumas, B. J. Anderson, J. P. Massey, and M. Wiedmann. 2006. Molecular epidemiology and cluster analysis of human listeriosis cases in three U.S. states. J. Food Prot. 69:1680-1689.

20. Schlech, W. F., III. 2000. Foodborne listeriosis. Clin. Infect. Dis. 31: 770-775.

21. Slutsker, L., and A. Schuchat. 1999. Listeriosis in humans, p. 7595. In E. T. Ryser and E. H. Marth (ed.), Listeria, listeriosis and food safety. Marcel Dekker, Inc., New York.

22. Swaminathan, B., and P. Gerner-Smidt. 2007. The epidemiology of human listeriosis. Microbes Infect. 9:1236-1243.

23. Vázquez-Boland, J. A., M. Kuhn, P. Berche, T. Chakraborty, G. Domínguez-Bernal, W. Goebel, B. González-Zorn, J. Wehland, and J. Kreft. 2001. Listeria pathogenesis and molecular virulence determinants. Clin. Microbiol. Rev. 14:584-640.

24. Wiedmann, M. 2002. Molecular subtyping methods for Listeria monocytogenes. J. AOAC Int. 85:524-531.

25. Wiedmann, M., J. L. Bruce, C. Keating, A. E. Johnson, P. L. McDonough, and C. A. Batt. 1997. Ribotypes and virulence gene polymorphisms suggest three distinct Listeria monocytogenes lineages with differences in pathogenic potential. Infect. Immun. 65: $\underline{2707-2716 .}$ 